\title{
Fusion17 summary talk: windsurfing on a cross section profile
}

\author{
Dieter Ackermann ${ }^{1, \star \star \star}$ and Lorenzo Corradi ${ }^{2, \star \star \star}$ \\ 1 GANIL - Bd Henri Becquerel BP 55027 - 14076 Caen Cedex 05 - France \\ ${ }^{2}$ INFN - Laboratori Nazionali di Legnaro - Viale dell'Universita' 2, 35020, Legnaro (Padova) - Italy
}

\begin{abstract}
The spectrum of physics, discussed in the $7^{\text {th }}$ edition of the "Fusion" conference series, included a number of topics, ranging from reaction dynamics to structure studies, from the lightest to the heaviest nuclei, from inelastic reactions to fusion and fission, with applications of astrophysical interest and the newest developments and findings in theory and experiment. With this paper we try to summarize the most relevant issues discussed during the five days of Fusion 17.
\end{abstract}

\section{Introduction}

Before starting our considerations, we wish to express our compliments to the organizers for their excellent job. We enjoyed very much the professional organization of the conference, the stimulating and friendly atmosphere and the high quality level of speakers and talks. Fusion17 was an excellent continuation of the good tradition of the "Fusion" conference series, where a wealth of important new experimental and theoretical work has always been at the focus of intense debate.

The history of conferences dealing with heavy ion collision at the Coulomb barrier had started with the first meeting in 1984 at MIT in Cambridge U.S.A. The first conference carrying "Fusion" in its name was organized in 1997 in South Darras, New South Wales - Australia. After stations in Matsushima Miyagi - Japan (2003), Venice Italy (2006), Chicago - U.S.A. (2008), Saint Malo - France (2011) and New Delhi - India (2014), it came back after exactly 20 years to Australia and Hobart on the Tasmanian island for the 2017 edition. In the following we report on the second experience "down under" and the rich and comprehensive program, trying to trace the relevant issues raised during the conference. Of course the choice of subjects reflects our personal view and taste. We felt it unnecessary to list specific figures or references, most of which will be found in the individual contributions to the proceedings.

\section{An overview}

In an attempt to establish the general frame and the main skeleton of fusion conferences, we can roughly distinguish three main energy domains, as shown in Fig. 1.

\footnotetext{
^e-mail: ackermann@ganil.fr

$\star \star$ the corresponding author was supported by the European Commission in the framework of the CEA-EUROTALENT program

$\star \star \star$ e-mail: corradi@lnl.infn.it
}
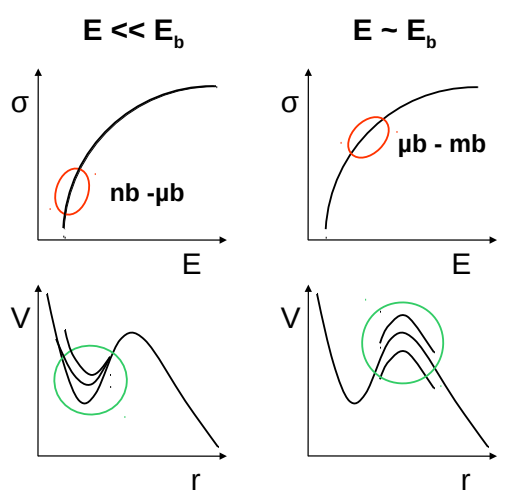

$\sigma$ steep fall off

structure of $V_{N}$ at $r<r_{B}$

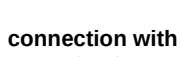

CC effects

$D(B)$ from fusion

and QE processes

connection with

connection with

surface vibrations and transfer
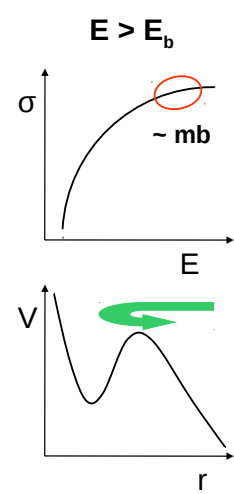

fusion "hindrance"

$\sigma$ fusion $<\sigma$ capture

connection with QF, MNT, DIC, BU

Figure 1. Schematic view of fusion reactions in terms of cross section and associated parts of the nuclear potential, based on bombarding energy ranges.

a) the energy range well below the Coulomb barrier, where cross sections undergo a steep fall off. Here one can explore the shape of the nuclear potential at distances significantly shorter than the fusion barrier radius. Moreover, this is, where a strong connection with astrophysics is established.

b) the energy range near the Coulomb barrier, where coupled channel effects due to surface vibrations and nucleon transfer show up. This is, where the concept of barrier distributions that one can extract from the excitation function for fusion or for quasi-elastic scattering, best applies.

c) the energy range above the Coulomb barrier, where fusion cross sections can be hindered, especially for heavy 


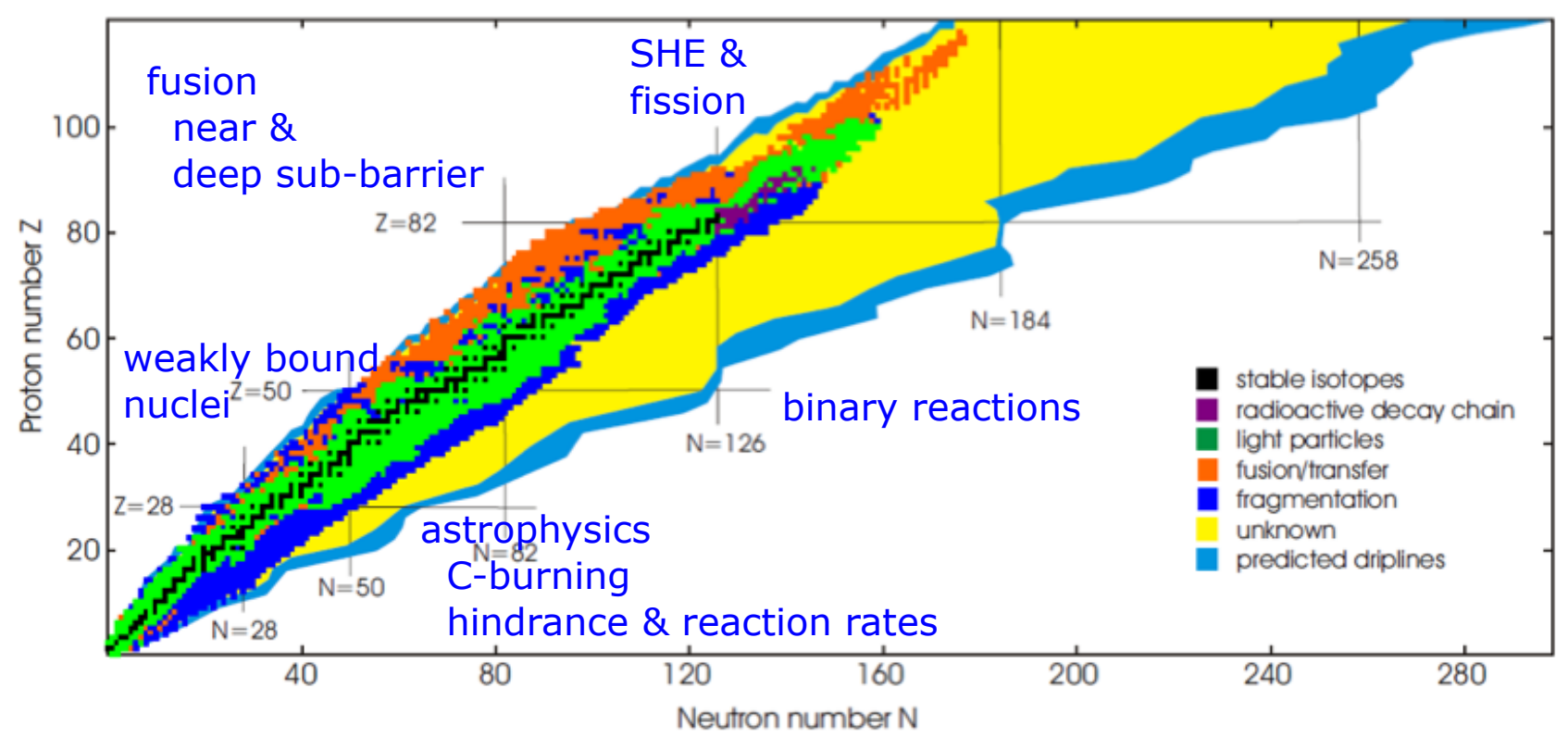

Figure 2. Chart of nuclides illustrating which nuclei have been discovered so far by the various production mechanisms listed in the agenda (courtesy of M. Thoennessen; Rep. Prog. Phys. 76 (2013) 056301). In addition the topics discussed during FUSION17 are indicated (in blue). They are partly localized in various areas of the nuclear chart like the superheavy element (SHE) research at the upper right part or the fusion reactions with weakly bound nuclei at the proton and neutron driplines, mainly in the low mass region. Others, such as binary reactions or the fusion barrier distribution studies, span almost the whole nuclear landscape.

systems. Here the fusion cross section is lower than the one for capture, as a consequence of the competing reaction channels like quasi-fission (QF), multinucleon transfer (MNT) and deep inelastic (DIC) or (especially for weakly bound nuclei) break-up (BU) processes.

Within this frame, many questions were debated and continue to be important issues in the field. Among these questions are the origin of the steep fall off of fusion cross sections, and the connection between fusion, binary processes and other reaction channels (e.g. break-up, especially for weakly bound systems). Challenges are the understanding of the dynamics of fusion reactions for very heavy nuclei, and how this can be quantitatively treated within microscopic theories. These questions were at the core of Fusion17, emerging through the various presentations in the different sub-fields explored during the conference, from astrophysics to fission dynamics and heavy element formation.

In Fig. 2 a compilation of the chart of nuclides is shown, illustrating which nuclei have been discovered through the different (reaction) mechanisms employed so far. Fusion and binary reactions are mainly aiming at nuclei located left from the valley of stability. However, more neutron rich species entered the focus of transfer studies recently with the employment of neutron rich reaction partners, like the region below ${ }^{208} \mathrm{~Pb}$ close to the $\mathrm{N}=126$ neutron shell closure. The extremes of the chart of nuclides in the lighter mass area close to the proton and neutron drip lines play a decisive role in reaction studies using weakly bound reaction partners. The topics discussed at Fusion17 summarized in this report are also indicated in Fig. 2, some of them referring to clearly localized re- gions like the SHE research and fission studies in the upper right part or the just mentioned weakly bound nuclei in the lower left part. Others are involved in investigations for which the whole chart of nuclides is relevant, like fusion barrier studies or binary reactions. The Fusion 17 contributions dealing with these topics will be summarized in the following sections.

\section{Heavy and superheavy elements}

Y. Aritomo, M. Block, Ch.E. Düllmann, J.M. Gates, J. Khuyagbaatar, E. Litvinova, W. Loveland, W. Mittig, P. Schwerdtfeger, J.R. Stone, T. Tanaka, Ch. Theisen, M. Thoennessen

The frame of the field of research concerning heavy and superheavy nuclei, but not only, was set by M. Thoennessen by presenting the trends of isotope production and element production as function of time. He was conveying a rather positive message showing a still linear growth of these numbers throughout the last 80 years. At the forefront of the superheavy element synthesis, one faces, however, extreme difficulties according to Ch.E. Düllmann, when trying to analyse the model predictions for synthesis cross sections for the next heavier elements to be synthesized with $Z=119$ and 120. Some way out of this was shown by J. Khuyagbaatar who presented the correlation between the minimum emission angle of fission fragments and the compound nucleus production for heavy systems, and by T. Tanaka who finds that the barrier distributions he extracts from quasi-elastic back scattering show the barrier positions at energies, where the maximum production 
cross sections are experimentally observed (see also section 5).

Y. Aritomo analysed, how his model predictions, based on the Langevin equation, depend on parameters like the fission barrier or friction for different systems, illustrating its range of applicability. While W. Loveland reminded of the importance of the compound nucleus formation probability for the successful evaporation residue creation for heavy systems, W. Mittig illustrated among other features the possibility of the production of superheavy nuclei in deep inelastic binary reactions of two very heavy colliding nuclei in the high $\mathrm{Z} /$ high A tail of the populated isotopic distribution, as proposed by V. Zagrebaev and W. Greiner.

In the last decade of the last century W. Greiner and others had suggested exciting features of atomic systems of very high atomic number, including the particle production from the vacuum, when negative energies for the inner shell electrons of the "super-atom" should be reached. They suggested, in particular, a limit for the highest possible $\mathrm{Z}$ related to the fine structure constant $\alpha=1 / 137$. P. Schwerdtfeger presented a critical analysis of this in the view of a possible end of the periodic table of Mendeleev, elaborating on features which would motivate an extension of this limit like e.g. screening effects. He also illustrated the state-of-the art approaches to this question by quantum chemical theory approaches, including P. Pykkö's predictions which are advertising 172 as the critical $\mathrm{Z}$.

The relativistic nuclear field theory approach extended by quasi particle vibrational coupling was shown by E. Litvinova to be capable of predicting single particle level properties, possible shell gaps and excitation spectra of superheavy nuclei in the region of the predicted island of shell stabilized superheavy nuclei (SHN), being bench marked for lighter species like doubly magic tins. J.R. Stone could show that her results, obtained by calculations employing quark-meson coupling for various nuclear properties like e.g. masses and binding energies, agreed well with the ones obtained by the finite range liquid droplet model by P. Möller.

The study of decay properties of the heaviest species will be pursued at the LBNL in the near future with the help of a new mass selection device, FIONA, as presented by J.M. Gates. Decay spectroscopy of the heaviest species in the region of deformed shell stabilized nuclei, from fermium up to darmstadtium isotopes, was also discussed by $\mathrm{Ch}$. Theisen who highlighted special nuclear structure features like K-isomers, being prevalent for these nuclei. M. Block illustrated with the example of the heaviest nuclei for which such investigations had been performed up to now, in the nobelium-lawrencium region, that atomic physics methods like laser spectroscopy represent a powerful alternative approach to nuclear structure properties like nuclear spin, magnetic and hence quadrupole moments, and isotopic shifts and hence changes in mean square charge radii.

\section{Fusion at near barrier energies}

R.T DeSouza, L. Guo, K. Hagino, H.M. Jia, Khushboo, C.J. Lin, K. Vo-Phuoc, P.D. Stevenson, K. Washiyama
$\mathrm{K}$. Hagino gave an overview of the recent studies on near and sub barrier fusion reactions, pointing out the present theoretical challenges and classifying the various employed theoretical models on the basis of the macroscopic or microscopic treatment. On one side we have mixed microscopic-macroscopic concepts, based for instance on coupled channels, which were quite successfull in the explanation of a large body of experimental data since one can incorporate inelastic and transfer degrees of freedom. On the other hand one aims at describing sub-barrier fusion within a fully microscopic frame. Microscopic theories like Time Dependend Hartree Fock (TDHF) are being used more and more extensively, especially in connection with the integration of other mechanisms, like deep inelastic or fission. However many problems need to be overcome, like the inclusion of nucleonnucleon correlations and, for fusion reactions, the missing treatment of tunnelling. Important, in general, is the need to strongly interconnect structure and dynamics, while different models often overemphasize only one of the two aspects.

R.T. DeSouza presented a comparison between fusion cross sections of ${ }^{18,19} \mathrm{O}+{ }^{12} \mathrm{C}$, and showed that for the more neutron rich (radioactive) ${ }^{19} \mathrm{O}$ case, the cross sections above the barrier are larger by $20 \%$, while below the barrier they are enhanced by a factor three compared with the ${ }^{18} \mathrm{O}$ case. This underlines the importance to properly take into account nuclear structure properties. Structure properties were also studied by Khushboo who presented new data on sub-barrier fusion of ${ }^{40} \mathrm{Ca}+{ }^{70} \mathrm{Zn}$ and ${ }^{28} \mathrm{Si}+{ }^{94} \mathrm{Zr}$ measured with the HIRA spectrometer of IUAC in New Delhi. She discussed how the change of deformation after neutron transfer can affect fusion.

H.M. Jia measured fusion cross sections for the ${ }^{32} \mathrm{~S}+{ }^{112-124} \mathrm{Sn}$ systems, where $\mathrm{Sn}$ isotopes have similar inelastic collective properties, and concluded that although one can find a correlation between transfer channels and fusion enhancements, the role of positive Q-value transfer channels is not yet understood. C.J. Lin showed new results on simultaneous measurements of fusion evaporation and fusion-fission cross sections for the ${ }^{32} \mathrm{~S}+{ }^{170} \mathrm{Er}$ and ${ }^{28} \mathrm{Si}+{ }^{174} \mathrm{Yb}$ systems, where capture cross sections have been reproduced by coupled channel and dinuclear system models.

Different theoretical talks focused on calculations based mainly on TDHF theory. L. Guo discussed the role of the tensor force in heavy-ion fusion, incorporating it into the TDHF frame and showing that it can increase the barrier height. This finding was partially corroborated by K. Washiyama who analyzed the effect of fusion hindrance near the barrier, proposing that the main contribution to the extra-push energy in heavy systems can possibly originate from a dynamical increase of the potential barrier. K. VoPhuoc discussed the effect of the excitation of vibrational states and of transfer on fusion within both static HF and TDHF, while P.D. Stevenson analysed the implications of effective interactions (Skyrme) on fusion dynamics near the barrier. 


\section{Fusion at deep sub-barrier energies}

\section{G. Colucci, F. Galtarossa, T. Ichikawa, C. Simenel, T. Tanaka}

Fusion studies at sub-Coulomb energies continue to be an interesting issue for two main reasons. The first is the not yet understood microscopic origin of the hindrance phenomenon and the second is their implications for nuclear reaction rates in astrophysically relevant sites. Which theory better suites for the explanation of experimental cross sections is still under debate. We should keep in mind that, when the position of the inner turning point becomes comparable to the touching point, the frozen density approximation may break down. As a matter of fact the predictions of the models based on a two-body frozen density approximation (coupled channels) and a one-body adiabatic approximation cannot be easily disentangled.

To deal with the hindrance phenomenon, T. Ichikawa discussed the importance of introducing in standard coupled channels a phenomenological damping factor, correlated to the reduced strength of the octupole vibration mode. C. Simenel showed the possibility to overcome the limitations of the TDHF theory, with no tunnelling effects, by incorporating the Pauli repulsion which may generate a proper potential well. G. Colucci and F. Galtarossa presented new cross section measurements at far sub-barrier energies in the ${ }^{28,30} \mathrm{Si}+{ }^{30} \mathrm{Si}$ and ${ }^{12} \mathrm{C}+{ }^{30} \mathrm{Si}$ systems, respectively, in the context of searching for hindrance effects related with positive Q-values for compound nucleus formation. The signature for hindrance, a maximum in the Sfactor, was observed for ${ }^{12} \mathrm{C}$ induced reaction but not for the $\mathrm{Si}+\mathrm{Si}$ systems.

T. Tanaka presented barrier distributions $\mathrm{D}(\mathrm{B})$ extracted from quasi-elastic backscattering, which were measured at the gas-filled separator GARIS of RIKEN for the ${ }^{48} \mathrm{Ca},{ }^{50} \mathrm{Ti}+{ }^{208} \mathrm{~Pb}$ and ${ }^{48} \mathrm{Ca}+{ }^{248} \mathrm{Cm}$ systems, which were used to synthesize heavy and superheavy isotopes with atomic numbers 102, 104 and 116, respectively. To accomplish this job it is important to properly separate the quasi-elastic from the deep-inelastic components, as already shown in previous work. Interestingly, the maxima of the $\mathrm{D}(\mathrm{B})$ correspond roughly to the energies of the maxima of the fusion cross sections for the various isotopes, a fact which requires some explanation (see also section 3 ).

\section{Reactions of astrophysical interest}

A. Caciolli, S. Courtin, M. La Cognata, T. Kawabata, R.G. Pizzone, K.E. Rehm, A. Wallner

The colourful world of nuclear astrophysics was introduced by K.E. Rehm who gave an overview over the possible locations and sites of the various stellar nucleosynthesis processes, pointing to the importance and the difficulties of deep sub-barrier fusion studies. Deep subbarrier fusion studies were then also the subject of many contributions to the conference ranging from the ${ }^{7} \mathrm{Li}$ problem discussed by T. Kawabata to the fusion reactions in medium-light systems presented by G. Colucci and F. Galtarossa (see also section 5), this last contribution providing an additional data point to the systematics established by C.L. Jiang.

Important for the stellar C-burning, new results on ${ }^{12} \mathrm{C}+{ }^{12} \mathrm{C}$ with consequences for stellar reaction rates and the observed ${ }^{26} \mathrm{Al}$ abundance in our Galaxy were presented by S. Courtin. For ${ }^{4} \mathrm{He}\left({ }^{8} \mathrm{Li}, \mathrm{n}\right){ }^{11} \mathrm{~B}, \mathrm{M}$. La Cognata observed a step like structure of the cross section, which he proposes to possibly relate to the partial wave contributions to the fusion excitation function. A. Caciolli reported on low energy fusion studies for the reactions ${ }^{17} \mathrm{O}(\mathrm{p}, \alpha){ }^{14} \mathrm{~N},{ }^{22} \mathrm{Ne}(\mathrm{p}, \gamma){ }^{23} \mathrm{Na}$ and ${ }^{2} \mathrm{H}(\mathrm{p}, \gamma){ }^{3} \mathrm{He}$ at the LUNA underground laboratory in the present LUNA400 configuration and with the upgraded accelerator LUNA MV (see also sections 10).

Based on the alternative approach using the Trojan Horse Method and a rare isotope beam (RIB) projectile, G. Pizzone discussed the reaction ${ }^{18} \mathrm{~F}(\mathrm{p}, \mathrm{a}){ }^{15} \mathrm{O}$ studied via the surrogate reaction ${ }^{18} \mathrm{~F}\left(\mathrm{~d}, \alpha^{15} \mathrm{O}\right) \mathrm{n}$.

As a last day highlight of the conference, the search for traces of radionuclides, stemming from stellar events like nearby super novae, in terrestrial deep sea archives were illustrated by A. Wallner.

\section{Reactions with weakly bound nuclei and cluster properties}

E.F. Aguilera, K.J. Cook, A. Di Pietro, D. Fabris, F. Gramegna, M. Hussein, S. Kalkal, J. Kolata, M. La Commara, M. Mazzocco, A. Mukherjee, M. Nakao, V.V. Parkar, V. Pseudo, B. Schuetrumpf, E.C. Simpson, G.L. Zhang,

Reactions involving weakly bound nuclei, mainly with $\mathrm{Li}, \mathrm{B}$ and Be projectiles on heavy targets, have been presented by many authors. A main issue was the role of break-up channels on the fusion process. Due to the many ways in which a break-up process may occur (elastic break-up, break-up followed by partial fusion etc.), disentangling the various components from other effects mimicking the same final partition is notoriously very difficult. Ambiguities in the data interpretation are often amplified with radioactive beams, due to low beam intensity. In spite of these difficulties many groups are actively working in the field and new data have been accumulated, allowing to make more quantitative comparisons with employed theoretical models.

J. Kolata critically reviewed reactions with ${ }^{8} \mathrm{~B}$, showing how break-up cross sections are reproduced by continuum-discretized coupled channels (CDCC) calculations, with the total fusion cross sections being enhanced near and below the barrier. S. Kalkal presented new data using beams of ${ }^{6} \mathrm{Li}$, discussing how the different break-up mechanisms, including resonant and non-resonant states, and their sensitivity to different targets, can be experimentally investigated. E.C. Simpson and K.J. Cook discussed new experiments, mainly in Li induced reactions, aiming at disentangling through angle-velocity correlations 
whether the break-up process which may involve short or long lived resonant states mainly occurs before or after the minimum distance between the interacting nuclei in Coulomb trajectories. An interesting outcome of these new studies is that the break-up alone cannot account for the observed suppression of complete fusion, as it depends critically on the lifetime.

V.V. Parkar, M. Hussein and G.L. Zhang presented work connecting the break-up process with incomplete and complete fusion reactions. M. Mazzocco and V. Pseudo showed results of cross section measurements performed with the radioactive ${ }^{7} \mathrm{Be}$ and ${ }^{11} \mathrm{Be}$ beams, respectively. A. Mukherjee discussed the barrier distributions derived from studies of ${ }^{7} \mathrm{Li}$ induced reactions, while E.F. Aguilera showed fusion and elastic scattering data for reactions in the ${ }^{6} \mathrm{Li}+{ }^{58} \mathrm{Ni}$ system, and $\mathrm{M}$. La Commara presented results of elastic scattering in ${ }^{8} \mathrm{~B}+{ }^{208} \mathrm{~Pb}$. A. Di Pietro underlined the importance to carefully take into account the effect of the ion energy loss in the target on correlating energy and fusion cross sections, especially with radioactive beams, where the low beam intensity may require to employ a stack target arrangement.

Light ion systems were also discussed in the context of cluster structures. M. Nakao discussed calculations of cluster structures in mirror cluster systems, while B. Schuetrumpf presented results, where localization and cluster structures are generated by calculations based on density functional theory. Cluster structures have been also discussed by D. Fabris and F. Gramegna through experiments on pre-equilibrium mechanisms performed with the GARFIELD set-up at LNL.

\section{Binary reactions}

E. Fioretto, A. Karpov, C. Parascandolo, D.C. Rafferty, G. Scamps, K. Sekizawa, S. Szilner, N. Wang, E. Williams

Binary reactions continue to be an important item in our field, due to the tight connection with sub-barrier fusion, to the possibility to learn about the degrees of freedom (single particle, pair or even cluster) acting in the transfer process and, more recently, to the peculiar mechanism leading to the production of neutron rich nuclei.

S. Szilner reported on sub-Coulomb studies with closed shell and superfluid systems performed with the PRISMA spectrometer, where the experimental probabilities of one and two nucleon transfer could be well reproduced by microscopic calculations. She stressed the importance to treat, on the same footing, structure and dynamics to compare experiment and theory in absolute scale and to learn about correlations. The problematic issue connected with the pair correlations is of current interest in ongoing research with radioactive beams, where for example the pairing interaction is expected to be significantly modified in nuclei with extended neutron distributions. The newly accumulated data are also attracting a renewed interest by theorists. The identification of the effects of the pairing interaction partially motivated the work presented in the talk by G. Scamps, who showed a new kind of calculations performed in the framework of models based on
TDHF + BCS theory. The effect of pairing correlations was discussed also by K. Sekizawa who presented the results of calculations performed within time dependent density functional theory incorporating superfluidity, suggesting how the particle flow induced by the phase difference between the colliding nuclei (analogue of the Josephson current) is originating from soliton excitations.

How the process evolves for the quasi-elastic to the deep inelastic regime is an issue, as it involves the amount of energy loss in the reaction. One should stress that dissipative processes play a critical role in channels like quasifission or deep inelastic (see also Fig. 1), which contribute significantly to the total reaction cross section and which must be taken into account when computing capture. New measurements of transfer cross sections have been shown by D.C. Rafferty, pointing to the gradual onset of energy dissipation, and by E. Williams who discussed new data on ${ }^{58} \mathrm{Ni}+{ }^{60} \mathrm{Ni}$, where deep inelastic and quasi-fission components were compared with TDHF. C. Parascandolo showed new results on the onset of the dynamical dipole mode, comparing two cases with different entrance channel mass asymmetry.

Energy dissipation has also important consequences for the onset of secondary processes. E. Fioretto discussed how the "heavy" partners produced in binary reactions are presently receiving peculiar attention. In fact, certain regions of the nuclear chart, like that below ${ }^{208} \mathrm{~Pb}$ (important also for astrophyisics) or in the actinides, can hardly be accessed by fragmentation or fission reactions. Here multinucleon transfer may be a suitable mechanism to approach those neutron rich areas. New data have been shown on the ${ }^{197} \mathrm{Au}+{ }^{130} \mathrm{Te}$ system, where from mass-mass correlations, obtained via a kinematic coincidence technique and employing PRISMA, one could get absolute cross sections of heavy transfer products, which were compared with the GRAZING model. Emphasis on the production yield of heavy transfer products has been put also in the talk of A. Karpov who discussed the predictions of models based on the Langevin equation. He showed the kind of agreement presently reached with the (scarce) available data. N. Wang presented an extension of the Quantum Molecular Dynamics model, originally developed for reactions in the Fermi regime, to the energy region close to the Coulomb barrier, where the main features of deep inelastic processes have been reproduced.

\section{Fission reactions}

\section{A. Bulgac, M. Caamaño, D. Hinde, J. Khuyagbaatar, W. Mittig, K. Nishio, E. Prasad, S. Pullanhiotan, B. Schuetrumpf, K. Sekizawa, A.S. Umar, S.G. Zhou}

With 'to fuse or not to fuse' S. Pullanhiotan introduced the fusion-fission competition in the entrance channel in heavy ion collisions between fusion and quasi-fission, and he reported on fission measurements at IUAC.

A quite substantial part of the conference was dedicated to fission and related issues both from the experimental as well as from the theoretical point of view. 
K. Nishio had employed nucleon transfer to induce fission for the projectile target combinations ${ }^{18} \mathrm{O}+{ }^{238} \mathrm{U},{ }^{237} \mathrm{~Np}$, resulting in a comprehensive body of data for a large number of isotopes from uranium to californium. In these investigations he succeeded in systematically studying features like fission barriers, multi chance fission and excitation energy dependent mass distributions. Mass angular distributions had been also systematically studied by D. Hinde. He was pointing out the importance of the "magicity grade" in the entrance channel, i.e. the total number of magic nucleon numbers of target and projectile, and the N/Z ratio matching, as well as he was discussing nuclear deformation and implications for SHN. Conclusions from fission fragment angular distribution studies relevant for compound nuclear formation and SHE synthesis were also presented by J. Khuyagbaatar (see also section 3).

Apart from the possibility to produce SHE in the high Z/high A tail of the isotopical distribution (see section 3), W. Mittig discussed features like ternary fission and the role of the magic $\mathrm{Z}=208$ in ${ }^{238} \mathrm{U}+{ }^{238} \mathrm{U}$ collisions, a classic reaction in the context of strong atomic Coulomb fields. Fission in inverse kinematics studied at instruments like the FRS and ALADIN at GSI induced by fragmentation or as photo-fission, or VAMOS at GANIL induced by multinucleon transfer, was presented by M. Caamaño. E. Prasad was looking for the role of fission modes, possibly governing fission in the $\mathrm{A}=200$ mass region and below.

Fission dynamics was studied using time dependent density functional theory by A.S. Umar, while S.G. Zhou was elaborating on fission barriers in the framework of multidimensionally-constrained covariant density functional theory, elucidating e.g. a third barrier in light actinides. Using an extension of energy density functional theory employing superfluidity, A. Bulgac demonstrated the dynamical development following proton and neutron densities towards fission of a heavy nuclear system like ${ }^{240} \mathrm{Pu}$, performing real time calculations. A similar idea was developed by K. Sekizawa in a time-dependent superfluid local density approximation, considering the superfluidity of nuclear matter including features like e.g. the nuclear Josephson effect (see also section 8). Using density functional theory B. Schuetrumpf localized nucleonic clusters to describe the density distribution inside the nucleus modelling fission also in a real time approach (see also section 7).

\section{Instrumentation}

\section{B.B. Back, D. Bazin, A. Caciolli, I.P. Carter, G. Fruet, J.M. Gates, Ch. Theisen}

New instrumentation and the respective research and development are mandatory for the progress of a field and some of them have been already mentioned like the new mass spectrometer FIONA at the BGS of LBNL presented by J.M. Gates (see section 3) or the upgrade of the underground laboratory LUNA MV illustrated by A. Caciolli (see section 6).

With STELLA a new tool for astrophysical studies being installed at the $4 \mathrm{MV}$ pelletron facility, Andromède, of the IPN Orsay in France was introduced by G. Fruet. The set-up is dedicated to study stellar nucleo-synthesis processes like C-burning, employing particle and photon detection with an annular Si detector and the scintillators from the FATIMA array. The rotating target used in first tests had been developed in collaboration with GANIL and will play a major role also in the new separatorspectrometer set-up $\mathrm{S}^{3}$ of GANIL's SPIRAL2 facility, discussed by $\mathrm{Ch}$. Theisen. The new set-up will open new opportunities for various research lines including SHN (see section 3), the region of $\mathrm{N}=\mathrm{Z}$ nuclei and nuclear astrophysics served by the high intensity beams from the new LINAC.

The new gas-filled separator AGFA designed among others also for SHN research is presently being put into operation at ANL. B.B. Back showed a compact ion-optical configuration which will be used for decay spectroscopy after separation, employing a comprehensive particle and photon detection set-up, as well as for in-beam nuclear structure studies in conjunction with the efficient $\gamma$ detection array GAMMASPHERE. A second device, presently under preparation at the physics division of ANL, is the Argonne In-flight Radioactive Isotope Separator AIRIS which is being developed to enhance the radioactive beam capabilities of the ATLAS facility.

The solenoidal exotic rare isotope separator SOLEROO developed for RIB investigations at ANU was presented by I.P. Carter, and the active target time projection chamber designed for a wide field of applications, using the rare isotope beams being available at the new FRIB facility at NSCL/MSU presently being started with ReA3, was discussed by D. Bazin.

\section{Concluding remarks}

An exciting and comprehensive program perfectly organized in a stimulating environment made this edition of the Fusion conference series a great success. Theoretical approaches were elucidated in all depth, experimental findings confronted with possible explanations in lively discussions and new instrumentation presented, based on most advanced technologies, all together creating a sound picture of the present status of a vivid field.

Apart from the intense scientific debate, the human aspect had not been forgotten. Two colleagues and friends, Paolo Gomes and Valery Zagrebaev, known for their engaged and pioneering contributions to the progress of the field, had left the community during the last years, way too early as many of us sustain. The organizers had honoured their memory by assigning two prizes, carrying their names, for the best presentations given by students or early career researchers.

To conclude, we wish to thank once more the organizers for their warm hospitality and great efforts which were the key of success for the conference. We would also like to thank all of our community for their excellent presentations and lively discussions.

See all of you at the next Fusion20 conference in Shizuoka! 